Check for updates

Cite this: RSC Adv., 2018, 8, 4204

Received 14th January 2018

Accepted 16th January 2018

DOI: $10.1039 / \mathrm{c} 8 \mathrm{ra00414e}$

rsc.li/rsc-advances

\section{Nanotextured Si surfaces derived from block- copolymer self-assembly with superhydrophobic, superhydrophilic, or superamphiphobic properties $\uparrow$}

\begin{abstract}
Agnieszka Telecka, ${ }^{a}$ Tao Li, ${ }^{\text {ab }}$ Sokol Ndoni ${ }^{\text {ac }}$ and Rafael Taboryski (D) *a
We demonstrate the use of wafer-scale nanolithography based on block-copolymer (BCP) self-assembly for the fabrication of surfaces with enhanced wetting properties. All classes of wetting behaviour derived from the same BCP nanolithography step are demonstrated. An in situ etch mask is defined by selfassembly of polystyrene (PS) and dimethylsiloxane (PDMS) domains to form a predominantly hexagonal array with pitch size $(72 \pm 3) \mathrm{nm}$. The subsequent branched processing scheme, exclusively employing dry chemistry and reactive ion etching (RIE), allows the fabrication of nanoholes, nanopillars, or high aspect ratio nano-hoodoo features (overhang profile structures) with a diameter below $100 \mathrm{~nm}$. The surfaces are finally functionalized with either hydrophobic surface chemistry by self-assembly from the precursor perfluorodecyltrichlorosilane (FDTS), or hydrophilic surface chemistry obtained by oxygen plasma treatment. The different texture and surface chemistry configurations are characterized with respect to their wetting properties with water, alkanes and organic oils. While, both nano-pillar and nano-hole surfaces feature excellent superhydrophobic properties with water contact angles (WCAs) exceeding $170^{\circ}$ and roll-off angles below $5^{\circ}$, only the nano-pillar surfaces exhibit convincing superhydrophilicity with WCAs below $5^{\circ}$. The repellency of low surface tension liquids known as amphiphobicity is demonstrated for the nano-hoodoo surfaces.
\end{abstract}

\section{Introduction}

The control of wettability of functional surfaces achieved by artificial nano-structures has high technological importance in various applications. Current examples of applications that rely on engineered surface wetting properties are: heat-transfer technologies, ${ }^{1-3}$ anti-ice and anti-frost surfaces, ${ }^{4-9}$ fieldvisibility in endoscopy, ${ }^{10}$ harvesting of sunlight in photovoltaic devices, ${ }^{11}$ adhesion properties, ${ }^{12}$ anti-fouling surfaces, ${ }^{13}$ drag reduction, ${ }^{14}$ anti-fogging surfaces, ${ }^{15}$ and a variety of other applications such as cell culturing and off-set printing. ${ }^{16}$

Various surface engineering methods have been proposed for the fabrication of either super wetting or completely repellent surfaces for given liquids. The most common example is the super water-repellent (superhydrophobic) self-cleaning surface known in nature from various plant leafs, most notably leafs of the lotus flower. ${ }^{17}$ Coating of an entirely flat surface with the

${ }^{a}$ Department of Micro- and Nanotechnology, Technical University of Denmark, DK-2800 Kongens Lyngby, Denmark. E-mail: rata@nanotech.dtu.dk

${ }^{b}$ Department of Electronic and Electrical Engineering, University College London, WC1E7JE London, UK

${ }^{c}$ Center for Nanostructured Graphene, CNG, Technical University of Denmark, DK2800 Kongens Lyngby, Denmark

$\uparrow$ Electronic supplementary information (ESI) available. See DOI: $10.1039 / \mathrm{c} 8 \mathrm{ra} 00414 \mathrm{e}$ lowest possible surface-energy chemistry consisting of hexagonally aligned closest packed $\mathrm{CF}_{3}$ groups, only gives rise to a water contact angle of $119^{\circ},{ }^{18}$ which is not high enough to shed water and qualify as a superhydrophobic surface. ${ }^{19}$ Hence, for a surface to be considered superhydrophobic, the low surface-energy chemistry must be combined with nanoscopic or microscopic surface texture such that the liquid can rest on a chemically heterogeneous surface comprised of the texture asperities and air pockets in the so-called Cassie-Baxter wetting state. ${ }^{20-24}$ This composite solid/air surface, is sometimes referred to as a plastron. ${ }^{25}$ With the exception of super-wetting surfaces made by means of photocatalytic activation of metal-oxides, ${ }^{26}$ fabrication methods for surfaces with enhanced wetting properties, typically involve steps of providing both the required surface chemistry and a surface texture. Such fabrication methods can be roughly divided into top-down methodologies requiring a pattern-design step for nano- and micro-lithography ${ }^{23,27-30}$ or bottom-up methodologies such as self-assembly of macro-molecular domains and colloids followed by selective reactive ion etching, ${ }^{31-37}$ random etching processes, ${ }^{22,38-40}$ tensile elongation of surface textures ${ }^{14,41}$ or coating with nanoparticles..$^{42}$ High-performance surfaces such as the self-healing, slippery, liquid-infused porous surface reported by Wong et al. ${ }^{43}$ may even employ combinations of various fabrication strategies. However, most of the proposed methods for the generation of such functional surfaces, allow only for fabrication of features in size ranges 
above the light diffraction limit $(\sim 200 \mathrm{~nm})$, thus limiting their potential applications in e.g. photovoltaics and for optical elements.

A superhydrophilic surface should preferably be completely non-repellent for water with a WCA smaller than $10^{\circ}$, allowing water to spread continuously over the surface to create a uniform film. ${ }^{44}$ A superhydrophobic surface, on the other hand, is usually characterized by having a water contact angle greater than $150^{\circ}$ and to enable water droplets easily to roll off the surface. ${ }^{45}$ The superhydrophobic surfaces are however often wetted by low surface tension liquids like alkanes and organic oils because surface reliefs with vertical or tapered side walls (taper angle with horizontal $\psi \geq 90^{\circ}$ ) only support a robust Cassie-Baxter state when the contact angle $\theta_{\mathrm{Y}}$ (Young's contact angle) for a corresponding flat surface with the same chemical composition is larger than the taper angle $\theta_{\mathrm{Y}} \geq \psi \cdot \cdot^{22,46-48}$ Low surface tension liquids usually fail to comply with this condition, and thus these liquids will impregnate the texture and end up in a wetting state commonly referred to as the Wenzel state. ${ }^{\mathbf{4 9}}$

To conserve the plastron property for low surface tension liquids, the structures must have inverse (undercut) topography with $\psi<90^{\circ}$, called "re-entrant" structures. ${ }^{46,50}$ When $\psi$ is lower than the Young contact angle of the deposited liquid, a convex meniscus shape will impose a capillary stop barrier for the liquid and block further impregnation of the texture. The resulting wetting state is best described as a metastable Cassie-Baxter state corresponding to a well-defined local minimum in the free energy. ${ }^{\mathbf{4 6 , 5 0 - 5 2}}$ Superamphiphobic surfaces that repel both water and low surface tension liquids are of interest due to important potential applications such as reduction of biofouling, ${ }^{53}$ antifingerprint films, ${ }^{54}$ and chemical shielding, ${ }^{55}$ just to mention a few examples. Microstructures with re-entrant curvature have been successfully fabricated by various methods including reverse nanoimprint lithography, ${ }^{56}$ photolithography followed by selective etching ${ }^{57}$ or reaction-diffusion steps, ${ }^{58,59}$ colloidal templating of nanoparticles, ${ }^{60}$ electrodeposition of fluoropolymers, ${ }^{61}$ spray coating of functionalized silica, ${ }^{62}$ or by sol-gel approaches. ${ }^{63,64}$

In this paper, we present a branched wafer scale, fabrication scheme yielding nanostructured Si surfaces with feature sizes below $100 \mathrm{~nm}$, with either superhydrophobic, superhydrophilic, or superamphiphobic properties based on two self-assembly methodologies; one for the generation of nano-textures and another for the self-assembly of strongly hydrophobic fluorocarbon groups on the surface. Further, we demonstrate how the profiles of the nanotextures can be controlled by reactive ion etching (RIE) to yield all three possible classes of taper angle, namely $\psi>90^{\circ}, \psi=0^{\circ}$, and $\psi<90^{\circ}$. The proposed fabrication of nanotextures is based on self-assembly of block-copolymer polystyrene (PS) and dimethylsiloxane (PDMS) domains to form a predominantly hexagonal array of cylindrical PS domains, ${ }^{33,65-68}$ whereas the hydrophobic and amphiphobic surface chemistry is obtained by a self-assembled monolayer from the precursor perfluorodecyltrichlorosilane (FDTS) molecules $^{22}$ yielding a Young contact angle for water of $\sim 111^{\circ}$. The superhydrophilic counterparts of the surfaces are obtained by simply omitting the FDTS coating and terminate the process by a standard oxygen plasma cleaning step.
Methods such as electron beam and colloidal lithography has been previously reported for fabrication of superoleophobic nanostructures, such as in the work of Zeniou et al. ${ }^{69}$ where they report ultra-high aspect ratio silicon nanowires with diameters down to $200 \mathrm{~nm}$. Whereas Checco et al. ${ }^{\mathbf{7 0}, 71}$ demonstrated the fabrication of vertical nano-pillars and tapered nano-cones by a block-copolymer approach, and subsequently rendered the nanostructured silicon surfaces superhydrophobic by immersion in a solution containing octadecyltrichlorosilane (OTS); we extend the catalogue of possible surface topographies by fabricating vertical sidewall pillars and holes, conical pillars, and nano-hoodoos (re-entrant profile pillars). ${ }^{72,73}$ Further, we demonstrate how these surfaces elegantly can be rendered either superhydrophobic, superhydrophilic, or superamphiphobic only using dry chemistry. We fabricated both nanoholes and nanopillars of various aspect ratios and taper angles, exhibiting apparent water contact angles $\theta$ ranging from less than $5^{\circ}$ to more than $160^{\circ}$, depending on post-treatment surface chemistry. By adding the second reactive etching step comprising a modified mixture of reactive gasses, it was possible to generate the nano-hoodoo surface features yielding repellency of both water and low surface tension liquids. To the best of our knowledge, nanotextures exhibiting all classes of wetting behavior derived from the same block-copolymer vapor annealing step have not yet been presented. In particular, the superamphiphobic property of a textured surface with hexagonal pitch size as low as $(72 \pm 3) \mathrm{nm}$ has not yet been demonstrated by any self-assembly fabrication method on wafer scale.

\section{Results and discussion}

\section{Fabrication}

The nanotextured surfaces were fabricated by BCP nanolithography employing the process schematically presented in Fig. 1. PS-PDMS block-copolymer was directly spun onto a silicon wafer (a) and annealed in an atmosphere of solvent vapours creating PS cylinder-domains forming a predominantly hexagonal array (b). Oxygen $\left(\mathrm{O}_{2}\right)$ plasma was applied to remove PS blocks and oxidize PDMS blocks, resulting in a robust in situ silicon oxycarbide ceramic mask for the subsequent pattern transfer (c). Shallow nanoholes in silicon were generated by reactive ion etching using chlorine $\left(\mathrm{Cl}_{2}\right)$ gas chemistry $(\mathrm{d})$. High aspect ratio nanoholes were fabricated at this step by optimizing the $\mathrm{Cl}_{2}$ etching time. By coating with a perfluorodecyltrichlorosilane (FDTS) self-assembled monolayer nanotextured surfaces appeared superhydrophobic. Nanopillars were fabricated through initial deposition of a thin alumina $\left(\mathrm{Al}_{2} \mathrm{O}_{3}\right)$ layer by atomic layer deposition (ALD) (e) on the shallow nanohole patterned substrate from step (d) that worked as an in situ etching mask for the subsequent etch. Conical- (f) and straight side-wall $(\mathrm{g})$ nanopillars were generated by $\mathrm{Cl}_{2}$ etching, following $\mathrm{Al}_{2} \mathrm{O}_{3}$ deposition. Due to the $\mathrm{Cl}_{2}$ etching rate difference between $\mathrm{Al}_{2} \mathrm{O}_{3}$ and $\mathrm{Si}$, a reversal of the nanohole relief was obtained. Varying nanostructure aspect ratios were achieved by adjustment of etching time. Consecutive $\mathrm{O}_{2}$ plasma treatment rendered the nanotextured surfaces superhydrophilic whereas additional FDTS coating resulted in superhydrophobic 


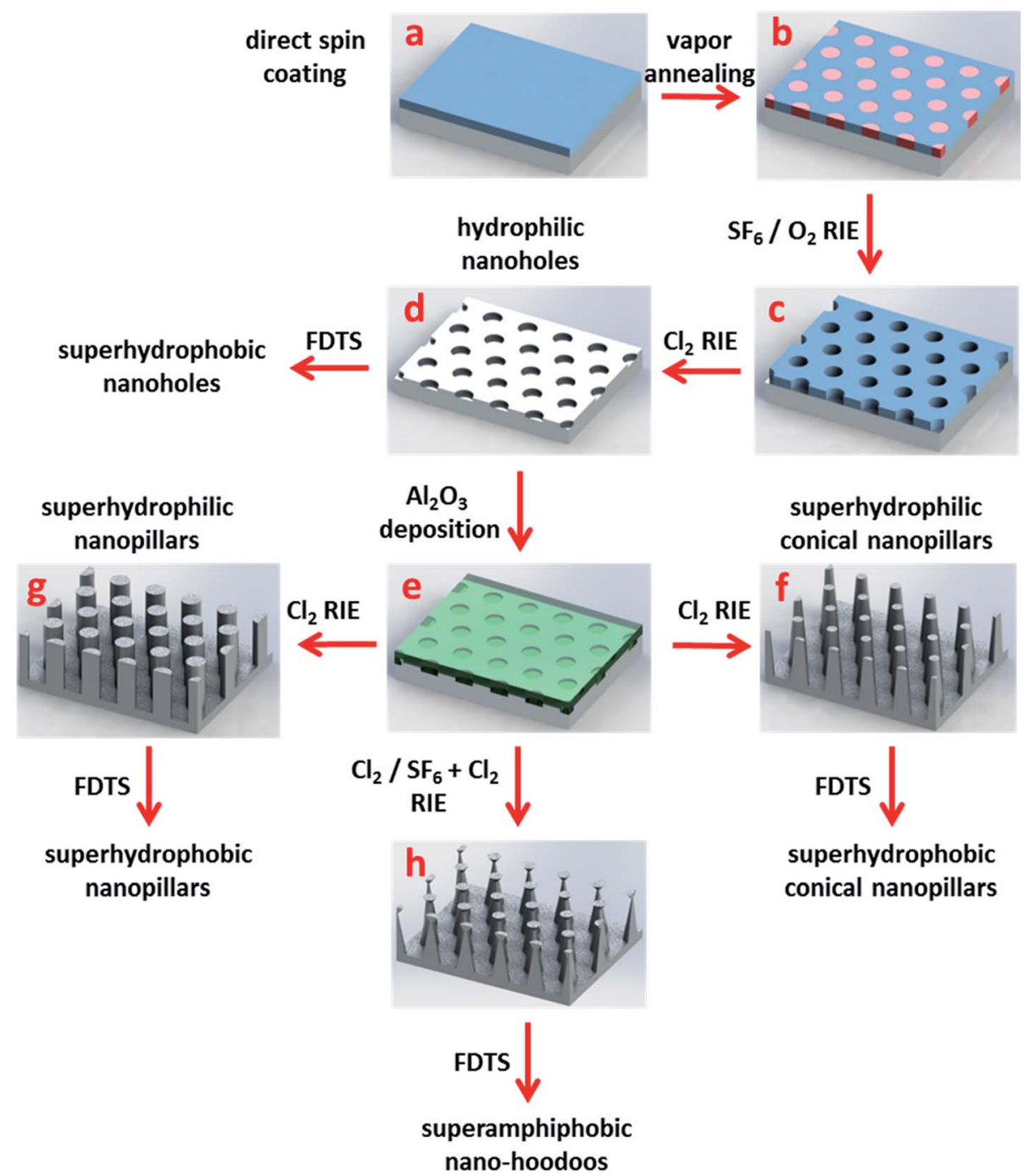

Fig. 1 (a) Spin coating of PS-PDMS block-copolymer. (b) Solvent annealing. (c) Oxygen plasma $\left(\mathrm{O}_{2}\right)$ removal PS blocks and oxidization of PDMS blocks. (d) Shallow nanoholes formed by etching by reactive ion etching in $\mathrm{Cl}_{2}$. (e) $\mathrm{Al}_{2} \mathrm{O}_{3}$ deposition. (f) Formation of high aspect ratio tapered nano-pillars. (g) Formation of straight side-wall nanopillars. (h) Over-hanging nanopillars generated by combined $\mathrm{Cl}_{2} / \mathrm{SF}_{6}+\mathrm{Cl}_{2}$ two-step etching.

surfaces. Nano-hoodoos were generated by a combined $\mathrm{Cl}_{2} / \mathrm{SF}_{6}$ $+\mathrm{Cl}_{2}$ two-step etching $(\mathrm{h})$. A reversal of the nanohole relief was obtained at the first $\left(\mathrm{Cl}_{2}\right)$ etching step. The addition of fluorine gas $\left(\mathrm{Cl}_{2}+\mathrm{SF}_{6}\right)$ during the second etching step allowed for a tuning of the nanopillars shape resulting in inversetrapezoidal $\mathrm{Al}_{2} \mathrm{O}_{3}$ features placed on top of the conical sub $\sim 200 \mathrm{~nm}$ high silicon nanopillars. Subsequent coating with FDTS resulted in the superamphiphobic surface. Details of the fabrication process are given in the Experimental section. Each of the topographies was always made in both a superhydrophobic version by the FDTS coating, and in a superhydrophilic version by $\mathrm{O}_{2}$ plasma cleaning as the respective last steps, by simply cleaving the wafers in two parts after the structure defining processes.

\section{Superhydrophobic and superhydrophilic surfaces}

Fig. 2 shows the scanning electron microscopy (SEM) images of the fabricated nano-pillar and nano-hole surfaces together with their respective water contact angles both with- and without the FDTS coating. Whereas the average pitch size for both holes and pillars was defined by the thermodynamics of the self-assembly process to $(72 \pm 3) \mathrm{nm}$, the depths and diameters of holes and pillars, and thereby their aspect ratios (ARs) and their diameter to pitch ratios $d / a$ for the nanostructures could be altered. In Fig. 2a we show the example of a nanohole surface. For nanohole surfaces, the AR could be tuned by roughly a factor of two from $2.26 \pm 0.06$ to $4.9 \pm 0.3$ whereas the $d / a$ could only be tuned moderately from $0.70 \pm 0.03$ to $0.77 \pm 0.08$ by adjustment of $\mathrm{Cl}_{2}$ gas etching time from $250 \mathrm{~s}$ to $500 \mathrm{~s}$ (see Fig. S1, Tables S1 and S2 in ESI $\dagger$ ). When coated with FDTS the nanohole surfaces exhibited impressively high WCAs in the range from $\sim 155^{\circ}$ to $\sim 170^{\circ}$ depending on $\mathrm{Cl}_{2}$ etch time, whereas without the FDTS coating, the WCA for nanohole surfaces dropped to $\sim 20^{\circ}$ as shown in Fig. $2 b$.

The results for (predominantly) straight and tapered sidewall nanopillars are presented in Fig. $2 \mathrm{c}-\mathrm{f}$ respectively. The 

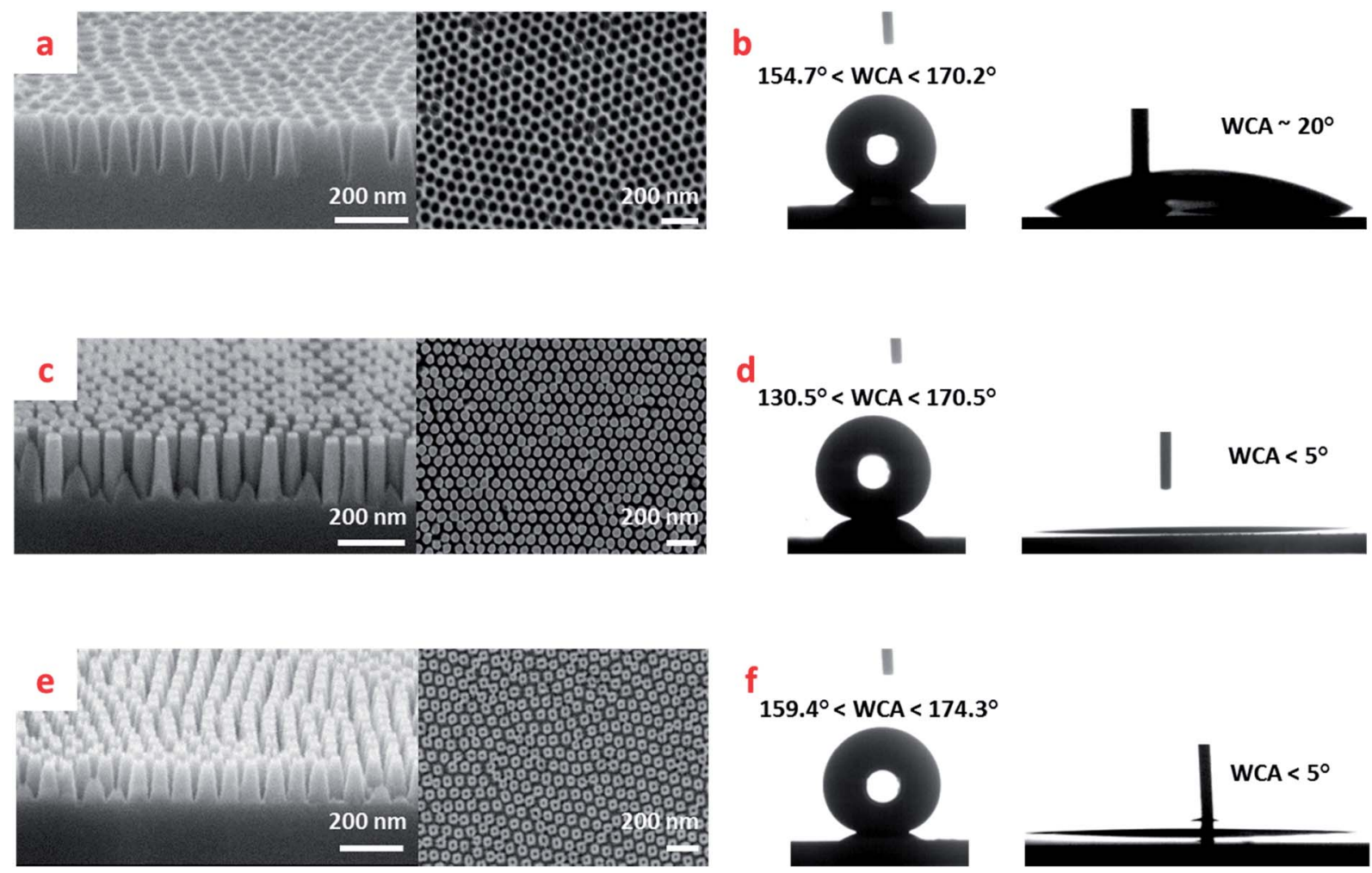

Fig. 2 (a) SEM images of nanoholes. (b) WCAs for the nanoholes in superhydrophobic and superhydrophilic states. (c) SEM images of straight side wall nanopillars. (d) WCAs for the straight side wall nanopillars in superhydrophobic and superhydrophilic states. (e) SEM images of tapered side wall nanopillars. (f) WCAs for tapered side wall nanopillars in superhydrophobic and superhydrophilic states.

tapered side-walls were obtained by deposition of a thinner $\mathrm{Al}_{2} \mathrm{O}_{3}(8 \mathrm{~nm})$ in situ mask than when fabricating the straight side-walls $\left(30 \mathrm{~nm}\right.$ ), followed by $\mathrm{Cl}_{2}$ gas etching. Varied pillar side slope angles were achieved through adjustment of etching time (80-120 s) to the mask thickness. For straight side-wall nanopillars, by increasing the $\mathrm{Cl}_{2}$ gas etching time within the range (170-320 s), a considerable increase in pillar height was obtained. In contrary to the nanohole surfaces, where the etching gas access and removal of reaction products from the bottoms of holes is impeded, the pillar surfaces were much more susceptible to AR and $d / a$ ratio tuning by etching time (see Table S2 in ESI $\dagger$ ). Hence, for the straight nano-pillar surfaces, tuning the $\mathrm{Cl}_{2}$ etching time in the interval (170-320) s resulted in ARs from $1.0 \pm 0.2$ to $13 \pm 2$ and $d / a$ decrease by roughly a factor of two from $0.9 \pm 0.1$ to $0.39 \pm 0.07$ (see Table S1 in ESI $\dagger$ ). The contact angle performance of the pillar surfaces is shown in Fig. 2d for the straight pillars, and in Fig. $2 \mathrm{f}$ for the conical pillars. We see that while superhydrophilic performance for both types of pillar surfaces is excellent with WCAs below $5^{\circ}$, the conical pillar surfaces exhibit slightly better superhydrophobic performance with WCAs in the range from $\sim 159^{\circ}$ to $\sim 174^{\circ}$ as compared to the straight pillar surfaces with WCAs ranging from $\sim 131^{\circ}$ to $\sim 171^{\circ}$ depending on $\mathrm{Cl}_{2}$ etching time.

In Fig. 3, we show the data for the obtained WCAs and roll-off angles for the pillar and hole surfaces when coated with the hydrophobic FDTS monolayer. The most important parameters are the diameter to pitch ratio $d / a$ and side slope angle of conical pillars. $d / a$ can be defined for both holes and pillars. Together with the side slope angle, $\beta$ this ratio determines the solid fraction $f_{\mathrm{s}}$ of the surface being wetted by the liquid as long as the sessile droplets remain in the Cassie-Baxter wetting state. In this respect, we see that the nanohole surfaces exhibit a strong water-repellant property also on tilted surfaces with roll-off angles $\alpha$ ranging from $7^{\circ}$ to $30.6^{\circ}$ as shown in Fig. 3b, with potential for self-cleaning surface applications. According to the Cassie-Baxter theory, ${ }^{20}$ the contact angle $\theta$ for a chemically heterogeneous surface comprised of solid and air, with solid fraction $f_{\mathrm{s}}$ can be calculated by:

$$
\cos \theta=-1+f_{\mathrm{s}}\left(1+\cos \theta_{\mathrm{Y}}\right) .
$$

For hexagonal pillar surfaces $f_{\mathrm{s}}$ can be expressed by $\left(\frac{\pi}{2 \sqrt{3}}\right)\left(\frac{d}{a}\right)^{2}$ and by $\left(1-\left(\frac{\pi}{2 \sqrt{3}}\right)\left(\frac{d}{a}\right)^{2}\right)$ for hole surfaces, where $d$ is the diameter of pillars/holes and $a=(72 \pm 3) \mathrm{nm}$ is the inter-pillar pitch size. $\theta_{\mathrm{Y}}$ is the Young's contact angle. For water on FDTS coated $\mathrm{Si}, \theta_{\mathrm{Y}}=(111 \pm 1)^{\circ}$. Eqn (1) is a special case of the theory, where it is assumed that no partial wetting of the structures occur, which is here expected to be well justified due to the small sizes and the dense nature of the texture features. For the same reason, we can expect the equation to be valid also for the hole surfaces. Fig. 3a presents the predicted CassieBaxter WCAs computed by using eqn (1) for the nanopillar and nanohole surfaces as a function of $d / a$ together with the 

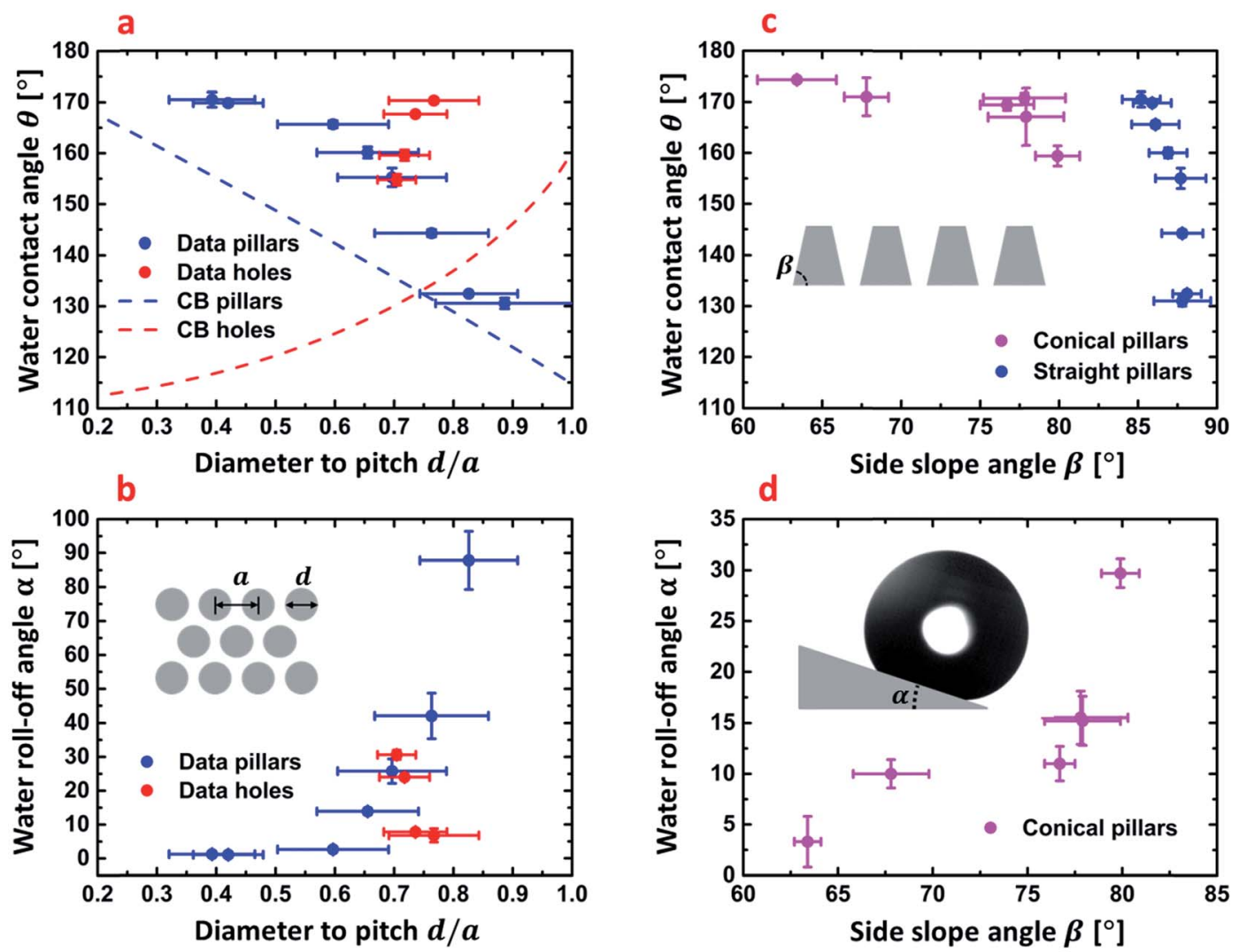

Fig. 3 (a) Water contact angles of straight side-wall nanopillars and nanoholes with calculated trend lines of by Cassie-Baxter equation (eqn (1)) as a function of diameter to pitch size ratio d/a ratio. (b) Measured roll-off angles of straight side-wall nanopillars and nanoholes as a function of the $d / a$ ratio, as defined in the inserted schematic. (c) Water contact angles of conical- and straight side-wall nanopillars as a function the side slope angle $\beta$ defined in the inserted schematic. (d) Measured roll-off angles of conical side-wall nanopillars as a function the side slope angle $\beta$. The inserted graphic shows the definition of the roll-off angle $\alpha$ as the inclination angle where the droplet start to roll on the surface uncertainties are obtained as SD ( $n=3$ ) (measured WCAs and roll-off angles) and from measurement of $d, a$ and $\beta$ by SEM inspection to determine SD ( $N=10$ ) and subsequent employment of standard rules for error propagation to determine uncertainties of $d / a$.

experimentally obtained values. We see that in terms of obtaining a high WCA and a low roll-off angle $\alpha$, both hole and pillar surfaces perform quite well, with a WCA exceeding $170^{\circ}$, and $\alpha$ getting well below $10^{\circ}$ for the best performing structures. The exact numbers are shown in Tables S1 and S2 in ESI. $\dagger$ The measured WCA values significantly exceed those calculated by eqn (1). This can be attributed the fact that the fabricated nanostructures may very well have additional protrusions on pillar tops and on hole walls. For the hole-surface this can clearly be seen to be the case in the profile view of Fig. 2a, where protrusions are seen at each triangular symmetry center. Such extra roughness will render the surface solid fraction $f_{\mathrm{s}}$ effectively smaller than estimated barely from diameters and pitch sizes. Moreover, shortcomings of the Cassie-Baxter equation to predict the exact apparent contact angles are well known and highly debated by the scientific community. ${ }^{27,74}$ For reference, we nevertheless include the plots based on eqn (1) using the nominal $d / a$ values. We notice that experimental WCA values increase with decreasing $f_{\mathrm{s}}$ for both hole and pillar surfaces which is in agreement with theoretical Cassie-Baxter prediction even though the measured WCAs are of by about $4-15 \%$ from the calculated values for pillar surfaces, and by about $15-40 \%$ for hole surfaces. Variation in $f_{\mathrm{s}}$ also influenced the mobility of deposited water droplets on titled surfaces as shown in Fig. 3b. The roll-off properties changed from an entirely pinned drop for a surface with nominal solid filling factor $f_{\mathrm{s}}=0.71$ to easy rolloff with $\alpha<1^{\circ}$ (the inclination angle required for the droplet to roll-off the surface) for the surface with $f_{\mathrm{s}}=0.13$. Fig. $3 \mathrm{c}$ shows how the water contact angle depends on the side slope angle $\beta$ for the conical pillar surfaces. The data for the predominantly straight pillar surfaces are plotted alongside for comparison. The trend indicate that the main effect of the sloping is a reduction in filling factor $f_{\mathrm{s}}$, when the pillars get pointier. Also, accordingly, the roll-off angle $\alpha$ decreases when the side slope angle $\beta$ decreases, as shown in Fig. $3 \mathrm{~d}$.

Now we turn to analyze the superhydrophilic properties of the nanostructured surfaces. The Young contact angle $\theta_{\mathrm{Y}}$ for a flat Si surface without FDTS coating, and terminated by a $10 \mathrm{~s}$ $\mathrm{O}_{2}$ plasma treatment was determined to $(22.9 \pm 0.6)^{\circ}$. In this case, the water will completely impregnate the surface texture, and the effective solid surface in contact with water will increase by the roughness factor $r(>0)$ defining the ratio of actual surface area to the projected surface area. According to a rigorous derivation by Whyman et al. ${ }^{21}$ Gibbs free energy for a spherical 
droplet resting on a hydrophilic textured surface forming the apparent contact angle $\theta$, can be written as:

$$
G=\gamma S-\pi(R \sin \theta)^{2} r \gamma \cos \left(\theta_{\mathrm{Y}}\right),
$$

where $S=2 \pi R^{2}(1-\cos (\theta))$ is the area of the liquid air interface, $R$ is the radius of the spherical droplet, $\gamma$ is the surface tension of the liquid, and $\theta_{\mathrm{Y}}$ is the Young contact angle. The Wenzel formula for the apparent contact angle is obtained by minimizing $G$, and yields: ${ }^{21,75}$

$$
\cos (\theta)=r \cos \left(\theta_{\mathrm{Y}}\right)
$$

Thus, for a roughness factor $r>1 / \cos \left(\theta_{\mathrm{Y}}\right), G$ will be a strictly increasing function of the contact angle in the interval $\theta \in\left[0^{\circ}\right.$; $\left.180^{\circ}\right]$, meaning that the system will attain the minimum energy for $\theta=0^{\circ}$ resulting in complete wetting of the surface. ${ }^{63}$ For hexagonal pillar- and hole-array surfaces, the roughness factor $r$ can readily be obtained from:

$$
r=\frac{\frac{1}{2} \sqrt{3} a^{2}+\pi d h}{\frac{1}{2} \sqrt{3} a^{2}}=\frac{\frac{1}{2} \sqrt{3}\left(\frac{a}{d}\right)^{2}+\pi\left(\frac{h}{d}\right)}{\frac{1}{2} \sqrt{3}\left(\frac{a}{d}\right)^{2}} .
$$

For straight sidewall nanopillars, calculated roughness factors, $r$ ranged from $\sim 4$ to $\sim 8$ (see Tables S1 and S2 in ESI $\dagger$ ), which clearly exceeds $1 / \cos \left(\theta_{\mathrm{Y}}\right) \approx 1.1$. The short exposure to $\mathrm{O}_{2}$ plasma rendered the surfaces superhydrophilic with WCA below $5^{\circ}$ for all nanotextures as shown in Fig. 2d. A similar superhydrophilic performance was observed for all tapered sidewall nanopillars as shown in Fig. 2f. The nano-hole surface on the other hand, which had even higher roughness factors, ranging from $\sim 6$ to $\sim 12$, did not exhibit similar superhydrophilic behavior with apparent WCA $\sim 20^{\circ}$ being only slightly below $\theta_{\mathbf{Y}} \sim 23^{\circ}$. We attribute this observation to capillary stopping effects during wetting of this type of surfaces, causing the system to attain a local minimum of the Gibbs free energy being higher than prescribed by eqn (2) and (4).

\section{Nano-hoodoos}

Nano-hoodoos $^{76}$ presented in Fig. 4a were fabricated by employing a two-step etching process following the $\mathrm{Al}_{2} \mathrm{O}_{3}$ layer deposition. While, an initial $\mathrm{Cl}_{2}$ gas etch, similarly to the previously described procedure, resulted in the nanopillar morphology, a subsequent etch with a $\mathrm{Cl}_{2}$ and $\mathrm{SF}_{6}$ gas mixture yielded the pattern schematically shown in Fig. $4 \mathrm{~b}$ and $\mathrm{c}$. The profile of the hoodoos can best be described as an oblate half ellipsoid with high eccentricity merged with an undercut inverted-trapezoid, which in turn is placed on the summit of a conical pillar. The convex texture angle $\psi$ is a crucial parameter that determines the re-entrant characteristics of the structure and can be calculated from the nano-texture geometry shown in Fig. 4c:

$$
\cos \psi=\frac{d-b}{2 c}
$$
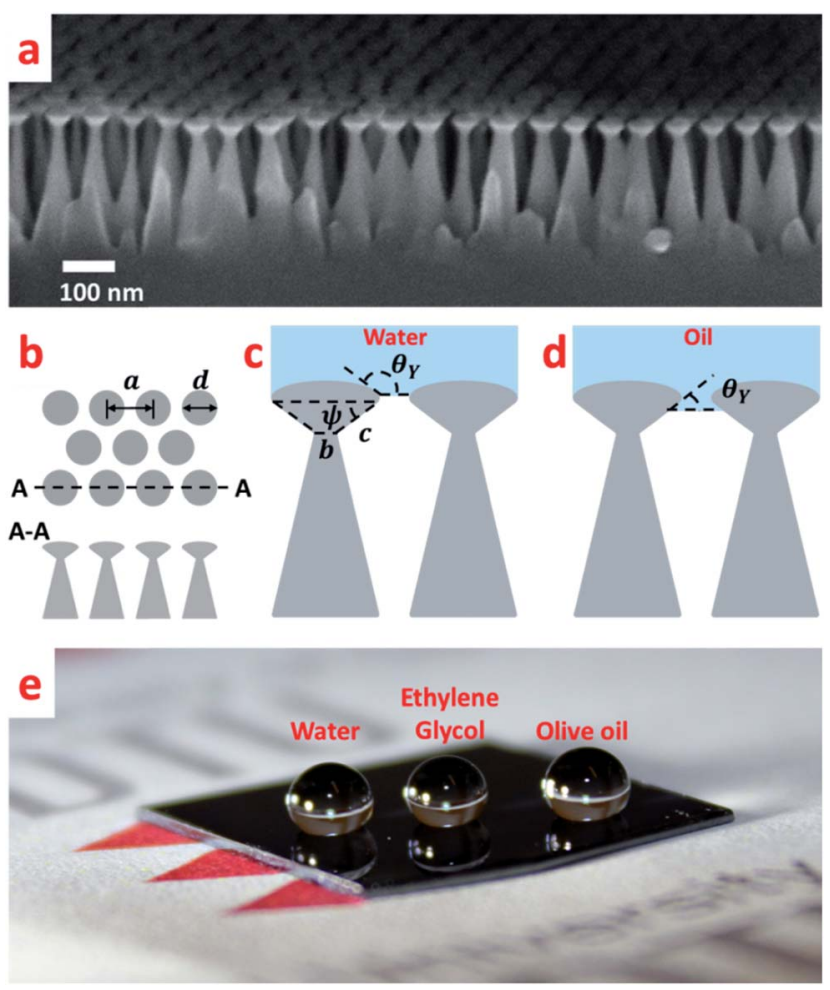

Fig. 4 (a) SEM image of BCP derived nano-hoodoos in profile view. (b) Sketch of nano-hoodoo array dimensions. (c) Sketch of inversetrapezoidal pillar profiles indicating geometric dimensions and convex texture angle $\psi$ showing wetting behavior with water. (d) Sketch of wetting behavior for low surface tension liquids. (e) Photography of sessile droplets of water $\left(72.8 \mathrm{mN} \mathrm{m}^{-1}\right)$, ethylene glycol $\left(47.7 \mathrm{mN} \mathrm{m}^{-1}\right)$ and olive oil $\left(32.3 \mathrm{mN} \mathrm{m}^{-1}\right)$ on nano-hoodoo Si surface coated with a monolayer of FDTS.

where $d$ and $b$ are the lengths of the parallel trapezoid feature bases, and $c$ is a convex side wall length. Table S3† lists the values of measured dimensions obtained from SEM Fig. 4a and S3 (see ESI $\dagger$ ). Using eqn (5), $\psi$ was determined to $(56 \pm 7)^{\circ}$ indicating the ability of the prepared surface to hold low surface tension liquids in a stable Cassie-Baxter state. ${ }^{46,77}$ The principle is sketched in Fig. 4c and d showing how the concave oblate ellipsoid part of the profile determines the wetting property with water, while the re-entrant trapezoid part of the profile prevents the low surface tension liquid from impregnating the texture. Impregnation of each liquid stops at the point, where the menisci change from concave to convex, i.e. when the contact angle to the profile matches the respective Young contact angle between the liquid and the material. ${ }^{46,50,51}$ The convex texture angle $\psi$ is a crucial but not sufficient condition for the structure to uphold low surface tension liquids in the stable Cassie-Baxter state. According to Tuteja et al., ${ }^{46}$ the appearance of the liquid-vapour interface sagging during liquid deposition, caused by body forces such as gravity, can be quantitatively measured for a specific structure by robustness parameters $H^{*}$ and $T^{*}$. These are the indicators of the robust composite interface formation, if $H^{*}$ and $\mathrm{T}^{*} \gg 1$. By modifying the Tuteja equations ${ }^{46}$ to fit our nanostructure geometry, 
Table 1 Apparent contact angles $\theta$ and roll-off angles $\alpha$ of liquids with varying surface tension $\gamma$ for nano-hoodoo FDTS coated surfaces. Images show the respective liquid droplets in profile view. All surface tensions, $\gamma$, were measured at $20^{\circ} \mathrm{C}$. Uncertainties are obtained as SD ( $n=3$ )

\begin{tabular}{lllll}
\hline & $\begin{array}{l}\text { Surface tension } \\
\text { Liquid }\end{array}$ & $\begin{array}{l}\text { Young's } \\
\text { contact angle } \theta_{\mathbf{Y}}\left[{ }^{\circ}\right]\end{array}$ & $\begin{array}{l}\text { Apparent contact } \\
\text { angle } \theta\left[\left[^{\circ}\right]\right.\end{array}$ & $\begin{array}{l}\text { Roll off angle } \\
\alpha\left[^{\circ}\right]\end{array}$ \\
\hline
\end{tabular}

Water

72.8

47.7

32.3

27.5

21.6
$110.9 \pm 0.6$

$87.0 \pm 0.5$

$83.10 \pm 0.04$

$70.0 \pm 0.4$

$63 \pm 2$
$161 \pm 7$

$152 \pm 3$

$155 \pm 3$

$140.4 \pm 0.3$

$38 \pm 2$

$107.8 \pm 0.9$

$21 \pm 3$

$34 \pm 3$

Pinned
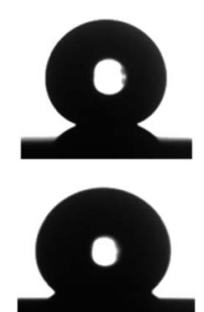

$63 \pm 2$

$n\left(\theta_{\mathbf{Y}}-\psi\right) /$

$H^{*}=\left(1-\cos \theta_{\mathrm{Y}}\right) r_{\mathrm{C}} l_{\text {cap }} /\left(\frac{(a-d)}{2}\right)^{2}$ and $T^{*}=l_{\text {cap }} \sin \left(\theta_{\mathrm{Y}}-\psi\right) /$
$(a-d)$, where $a$ is the average pitch size, $d$ is the feature diameter, and $r_{\mathrm{C}}$ is the radius of curvature of the oblate half ellipsoid, we calculated representative values of $H^{*} \approx 4 \times 10^{4}$ and $T^{*} \approx 2 \times 10^{4}$ respectively. In the numerical estimation of $H^{*}$, we used an estimated value of $r_{\mathrm{C}} \sim 5 \mathrm{~nm}$. Both parameters significantly exceed unity and are between one and four orders of magnitude higher then those previously reported for micrometer structures. ${ }^{50}$ According to this analysis, the presented nanohoodoo structure is expected to render the omniphobic surface extremely robust towards liquid impact. This property of the texture, originates from the much smaller feature sizes (nanometer scale) and very dense feature arrangement, which effectively limits the possibility of liquid sagging due to e.g. gravity effects. To maximize the contact angle formed with both water and hydrocarbon liquids, the nanostructure was coated with the FDTS monolayer which effectively reduces the surface energy of the Si surface. Fig. 4e shows a photo of water (with $\gamma=72.8 \mathrm{mN}$ $\left.\mathrm{m}^{-1}\right)$, ethylene glycol $\left(47.3 \mathrm{mN} \mathrm{m}^{-1}\right)$ and olive oil $\left(32.3 \mathrm{mN} \mathrm{m}^{-1}\right)$ droplets sitting on the FDTS coated nanotextured surface with the contact angles well above $150^{\circ}$ in all cases. Table 1 collects the values of Young contact angles $\theta_{\mathbf{Y}}$ and apparent contact angles of all tested liquids, measured on flat and nano-hoodoo surface, in all the cases with FDTS surface coating. For all liquids, the condition $\theta_{\mathrm{Y}}>\psi$ was accomplished as $\theta_{\mathrm{Y}}$ for octane, hexadecane, ethylene glycol, olive oil and water was measured to $63^{\circ}, 70^{\circ}, 83^{\circ}, 87^{\circ}$ and $111^{\circ}$ respectively. The nano-hoodoo surface exhibited gradually decreasing amphiphobic performance for tested liquids, in line with the surface tension $(\gamma)$ and measured $\theta_{\mathrm{Y}}$ values. A stable Cassie-Baxter state was achieved for olive oil, ethylene glycol and water, with an apparent contact angle $\theta$ approaching $160^{\circ}$. For hexadecane, characterized by significantly lower surface tension $\left(27.6 \mathrm{mN} \mathrm{m}^{-1}\right)$ and Young contact angle $\theta_{\mathrm{Y}}=70^{\circ}$, the nanotextured surface displayed an apparent contact angle $\theta$ of $140^{\circ}$. This is supported by the images of the droplets recorded during contact angle measurements, presented in the same table. For octane, the nano-hoodoo surface exhibit a contact angle $\theta$ of $107^{\circ}$ indicating that the liquid impregnates the texture to a higher degree and forms a metastable state which in nature is known from rose petals. ${ }^{23,78-81}$ This behavior is attributed to the proximate values of the octane Young contact angle of $\theta_{\mathrm{Y}}=(63 \pm 2)^{\circ}$ and the nanotexture angle $\psi=(56 \pm 7)^{\circ}$. Hence, $\theta_{\mathrm{Y}} \sim \psi$ when considering the standard deviations of both values. Nevertheless, this superamphiphobic nanotextured surface shows a remarkable stability against wetting transitions for hexadecane, olive oil, ethylene glycol and water during dynamic contact angle measurements and exhibits roll-off angles $\alpha$ of $38^{\circ}, 34^{\circ}, 21^{\circ}$ and $22^{\circ}$, respectively as quantitatively presented in Table 1 . Roll-off angles in the measured range indicate partial wetting of the nanostructures, especially for lower surface tension liquids as hexadecane and olive oil, but the ability of the surfaces to uphold the droplets during tilting with successful roll-off supports the hypothesis that despite the meager liquid sinking, the droplets remained in a Cassie-Baxter state.

\section{Conclusions}

We demonstrated the superhydrophobic, superhydrophilic and superamphiphobic effects for nanostructured surfaces derived 
from the same in situ etch mask comprising a hexagonal array with a pitch size well below the diffraction limit of visible light ( $200 \mathrm{~nm}$ ), which is important for e.g. anti-fogging and photovoltaic applications. The structures were synthesized on wafer scale without the use of any photo-masks or electronbeam writing. Instead, we employed a nanolithographic method based on the block-copolymer self-assembly combined with selective reactive ion etching steps, i.e. without using any wet chemistry. This approach considerably increases the overall process feasibility and process yield and allows for a fast and cheap patterning of in principle arbitrary large surface areas with a versatile hexagonal pattern, only requiring a homogenous application of the BCP solution and subsequent solvent annealing. By exploiting this process, we obtained nanostructures with varied morphologies; nanoholes and nanopillars, exhibiting exceptional superhydrophobic and superhydrophilic behaviours with WCAs reaching $170^{\circ}$ or less than $5^{\circ}$, depending on post-treatment (FDTS coating or oxygen plasma treatment). The hole-morphology may prove to have an advantage over the pillar morphology by having higher abrasion resistance due to the interconnection of the texture. ${ }^{82}$ Further, by modifying the etching process with the addition of another reactive gas $\left(\mathrm{SF}_{6}\right)$, we fabricated sub-200 $\mathrm{nm}$ high nano-hoodoos comprising inverse trapezoidal texture angle $\psi<90^{\circ}$ featuring repellency of low surface tension liquids. The nano-hoodoo surfaces could successfully hold water, ethylene glycol and olive oil in a stable Cassie-Baxter state, with apparent contact angles $\theta$ well above $150^{\circ}$ and roll-off angles below $35^{\circ}$. To the best of our knowledge, nanotextures exhibiting all classes of wetting behavior derived from the same block-copolymer vapor annealing step have not yet been presented. In particular, the superamphiphobic property of a textured surface with hexagonal pitch size as low as (72 \pm 3$) \mathrm{nm}$ has not yet been demonstrated by any self-assembly fabrication method on wafer scale.

\section{Experimental details}

\section{Materials}

Poly(styrene- $b$-dimethylsiloxane) (PS- $b$-PDSSMS) copolymer (SD 24) used for nanotexture fabrication was synthesized by an anionic polymerization following already reported procedure. ${ }^{\mathbf{8 3}}$

\section{Fabrication}

SD 24 dissolved in cyclohexane (0.25 wt\%) was directly spin coated (LabSpin6, SÜSS) on a silicon wafer yielding a thickness of $\sim 28 \mathrm{~nm}$ at a spinning speed of $2000 \mathrm{rpm}$. Solvent vapour annealing to direct densely packed PS cylinders into a hexagonal array was done at room temperature with the sample in a closed glass jar in the presence of the solvent vapour from methylcyclohexane for $160 \mathrm{~min}$. The in situ mask fabrication was performed in two etching steps using an Advanced Silicon Etcher (STS MESC Multiplex ICP serial no. 30343). For pretreatment, $\mathrm{SF}_{6}$ plasma was applied for $15 \mathrm{~s}\left(20 \mathrm{sccm} \mathrm{SF}_{6}, 20\right.$ mTorr, $50 \mathrm{~W}$ of coil power and $0 \mathrm{~W}$ of platen power) to remove the PDMS residual layer created on a top of the annealed surface and subsequent $\mathrm{O}_{2}$ plasma etching for $11 \mathrm{~s}\left(10 \mathrm{sccm} \mathrm{O}_{2}, 5\right.$
mTorr, $200 \mathrm{~W}$ coil power and $20 \mathrm{~W}$ of platen power) to remove PS blocks and oxidize PDMS blocks simultaneously, resulting in a hard silicon oxycarbide in situ mask of hexagonally arranged holes with high selectivity. The silicon etch was performed using ICP Metal Etch (SPTS serial no. MP0637) with breakthrough step conditions: $20 \mathrm{sccm} \mathrm{SF}_{6}, 3 \mathrm{mTorr}, 100 \mathrm{~W}$ of coil power and $10 \mathrm{~W}$ of platen power, for $35 \mathrm{~s}$, and following $\mathrm{Cl}_{2}$ etching: $20 \mathrm{sccm} \mathrm{Cl}$, $3 \mathrm{mTorr}, 300 \mathrm{~W}$ of coil power and $40 \mathrm{~W}$ of platen power, for $20 \mathrm{~s}$. For nanohole-surface fabrication, $\mathrm{Cl}_{2}$ etching step was extended to 250-500 s. For straight nanopillars and nano-hoodoos, an initial $30 \mathrm{~nm} \mathrm{Al}_{2} \mathrm{O}_{3}$ layer was deposited on a patterned substrate by ALD, working as an etching in situ mask for the subsequent etching. For tapered nanopillars, an initial $\mathrm{Al}_{2} \mathrm{O}_{3}$ layer of only $8 \mathrm{~nm}$ was deposited. ALD deposition was performed in Picosun ALD (model R200), using alternating exposures to trimethylaluminium (97\%, Aldrich) and deionized water $\mathrm{H}_{2} \mathrm{O}$ at $200{ }^{\circ} \mathrm{C}$ for 300 cycles with $\mathrm{N}_{2}$ gas purge steps in between. The exposures and purge times for both precursors used in this study were 0.2 and $25 \mathrm{~s}$, respectively. $\mathrm{Cl}_{2}$ etching (20 sccm $\mathrm{Cl}_{2}, 3 \mathrm{mTorr}, 300 \mathrm{~W}$ of coil power and $40 \mathrm{~W}$ of platen power) allowed for straight and tapered nanopillar fabrication with tuned aspect ratio by adjusting of etching time (170-320 s for straight nanopillars, 80-120 s for tapered nanopillars). Twostep $\mathrm{Cl}_{2} / \mathrm{Cl}_{2}+\mathrm{SF}_{6}$ etching (20 sccm Cl $2,180 \mathrm{~s}, 3 \mathrm{mTorr}, 300 \mathrm{~W}$ of coil power and $40 \mathrm{~W}$ of platen power; $20 \mathrm{sccm} \mathrm{Cl}_{2}, 30 \mathrm{~s}, 3 \mathrm{mTorr}$, $300 \mathrm{~W}$ coil power, $40 \mathrm{~W}$ platen power and $2 \mathrm{sccm} \mathrm{SF}_{6}, 30 \mathrm{~s}, 3$ mTorr, $300 \mathrm{~W}$ coil power, $40 \mathrm{~W}$ platen power) yielded the nanohoodoos surface. Nanostructures were coated with a precursor perfluorodecyltrichlorosilane (FDTS) self-assembled monolayer (MVD100E, Applied Microstructures Inc.) to yield superhydrophobic surfaces, or $\mathrm{O}_{2}$ plasma post-treated (ICP Metal Etch, SPTS serial no. MP0637; conditions: $40 \mathrm{sccm}, 10 \mathrm{~s}, 3$ mTorr, $200 \mathrm{~W}$ coil power, $20 \mathrm{~W}$ platen power) to yield superhydrophilic surfaces.

\section{Characterization}

Nanoscopy images of the nanostructures were taken by a Field Emission Zeiss Supra 40VP Microscope. Dimensions of nanopillars were measured by ImageJ $1.47 \mathrm{t}$ software. Contact angles (CA) were measured using an optical tilting cradle tensiometer (Attension Theta optical tensiometer, Biolin Scientific Holding $\mathrm{AB}$, Sweden) equipped with a high-speed camera (3000 fps, MotionXtra N3, IDT, USA) by the sessile drop method in static mode. The roll-off angle $\alpha$ was measured by the tilting plate method. Shapes of droplets were fitted by the Young-Laplace fitting method (Attention Theta software, version 4.2). For all contact angle measurements, droplets of $6.5 \mu \mathrm{l}$ volume were dispensed. The baseline was determined using an auto baseline function in Attention Theta software. The tensiometer cradle was tilted at $1^{\circ} \mathrm{s}^{-1}$ while capturing images of drop profiles with 1 frame per second. For each measurement, a sequence of images was obtained. Static contact angle was acquired from the first captured frame and the roll-off angle from the last frame before the drop completely rolled off from the surface. For each liquid three recordings were made with droplets dispensed on different areas. 


\section{Author contributions}

AT and RT conceived the project. TL and SN developed the BCP process. AT performed the experiments and characterization. AT made the data analysis. AT and RT wrote the manuscript with comments from all authors. Approval of the final version was given by all authors.

\section{Conflicts of interest}

The authors declare no competing financial interests.

\section{Acknowledgements}

DTU Danchip is acknowledged for clean room instrument training, and for the operation of a world-class clean room facility. This work was supported by the Technical University of Denmark, through a special PhD stipend for AT for collaboration with partner universities. T. L. and S. N. acknowledge support by the Center for Nanostructured Graphene sponsored by the Danish National Research Foundation, Project DNRF103.

\section{References}

1 N. Miljkovic, R. Enright and E. N. Wang, ACS Nano, 2012, 6, 1776-1785.

2 C. S. Sharma, J. Combe, M. Giger, T. Emmerich and D. Poulikakos, ACS Nano, 2017, 11, 1673-1682.

3 Y. M. Hou, M. Yu, X. M. Chen, Z. K. Wang and S. H. Yao, ACS Nano, 2015, 9, 71-81.

4 P. Kim, T. S. Wong, J. Alvarenga, M. J. Kreder, W. E. AdornoMartinez and J. Aizenberg, ACS Nano, 2012, 6, 6569-6577.

5 J. Y. Lv, Y. L. Song, L. Jiang and J. J. Wang, ACS Nano, 2014, 8, 3152-3169.

6 J. B. Boreyko and C. P. Collier, ACS Nano, 2013, 7, 1618-1627.

7 K. R. Murphy, W. T. McClintic, K. C. Lester, C. P. Collier and J. B. Boreyko, ACS Appl. Mater. Interfaces, 2017, 9, 2430824317.

8 P. Guo, Y. M. Zheng, M. X. Wen, C. Song, Y. C. Lin and L. Jiang, Adv. Mater., 2012, 24, 2642-2648.

9 L. Mishchenko, M. Khan, J. Aizenberg and B. D. Hatton, Adv. Funct. Mater., 2013, 23, 4577-4584.

10 S. Sunny, G. Cheng, D. Daniel, P. Lo, S. Ochoa, C. Howell, N. Vogel, A. Majid and J. Aizenberg, Proc. Natl. Acad. Sci. U. S. A., 2016, 113, 11676-11681.

11 J. Li, J. Zhu and X. F. Gao, Small, 2014, 10, 2578-2582.

12 K. Koch, B. Bhushan, Y. C. Jung and W. Barthlott, Soft Matter, 2009, 5, 1386-1393.

13 S. H. Ma, Q. Ye, X. W. Pei, D. Wang and F. Zhou, Adv. Mater. Interfaces, 2015, 2(13), 1500257.

14 M. N. Kavalenka, F. Vuelliers, S. Lischker, C. Zeiger, A. Hopf, M. Roehrig, B. E. Rapp, M. Worgull and H. Hoelscher, ACS Appl. Mater. Interfaces, 2015, 7, 10651-10655.

15 Z. Q. Sun, T. Liao, K. S. Liu, L. Jiang, J. H. Kim and S. X. Dou, Small, 2014, 10, 3001-3006.

16 E. Ueda and P. A. Levkin, Adv. Mater., 2013, 25, 1234-1247. 17 W. Barthlott and C. Neinhuis, Planta, 1997, 202, 1-8.
18 T. Nishino, M. Meguro, K. Nakamae, M. Matsushita and Y. Ueda, Langmuir, 1999, 15, 4321-4323.

19 K. S. Liu, X. Yao and L. Jiang, Chem. Soc. Rev., 2010, 39, 32403255.

20 A. B. D. Cassie and S. Baxter, Trans. Faraday Soc., 1944, 40, 0546-0550.

21 G. Whyman, E. Bormashenko and T. Stein, Chem. Phys. Lett., 2008, 450, 355-359.

22 L. Schneider, M. Laustsen, N. Mandsberg and R. Taboryski, Sci. Rep., 2016, 6, 21400.

23 E. Sogaard, N. K. Andersen, K. Smistrup, S. T. Larsen, L. Sun and R. Taboryski, Langmuir, 2014, 30, 12960-12968.

24 N. K. Mandsberg and R. Taboryski, Surf. Topogr.: Metrol. Prop., 2017, 5(2), 024001.

25 N. J. Shirtcliffe, G. McHale, M. I. Newton, C. C. Perry and F. B. Pyatt, Appl. Phys. Lett., 2006, 89(10), 104106.

26 R. D. Sun, A. Nakajima, A. Fujishima, T. Watanabe and K. Hashimoto, J. Phys. Chem. B, 2001, 105, 1984-1990.

27 S. T. Larsen and R. Taboryski, Langmuir, 2009, 25, 12821284.

28 N. K. Mandsberg and R. Taboryski, Langmuir, 2017, 33, 5197-5203.

29 M. Laurenti, A. Verna, M. Fontana, S. Stassi, G. Canavese, S. L. Marasso and V. Cauda, Adv. Mater. Interfaces, 2016, 3(13), 1600110.

30 E. Ueda, W. Q. Feng and P. A. Levkin, Adv. Healthcare Mater., 2016, 5, 2646-2654.

31 A. Dev, B. D. Choudhury, A. Abedin and S. Anand, Adv. Funct. Mater., 2014, 24, 4577-4583.

32 M. Luo and T. H. Epps, Macromolecules, 2013, 46, 7567-7579.

33 T. Li, Z. L. Wang, L. Schulte and S. Ndoni, Nanoscale, 2016, 8, 136-140.

34 H. T. Yang, X. Dou, Y. Fang and P. Jiang, J. Colloid Interface Sci., 2013, 405, 51-57.

35 T. Kraus, D. Brodoceanu, N. Pazos-Perez and A. Fery, Adv. Funct. Mater., 2013, 23, 4529-4541.

36 P. Kothary, X. Dou, Y. Fang, Z. X. Gu, S. Y. Leo and P. Jiang, J. Colloid Interface Sci., 2017, 487, 484-492.

37 S. Han, S. Ji, A. Abdullah, D. Kim, H. Lim and D. Lee, Appl. Surf. Sci., 2018, 429, 244-252.

38 E. Yu, S. C. Kim, H. J. Lee, K. H. Oh and M. W. Moon, Sci. Rep., 2015, 5, 9362.

39 J. U. Kim, S. Lee and T. I. Kim, J. Nanomater., 2016, 7602395. 40 R. Di Mundo, R. d'Agostino and F. Palumbo, ACS Appl. Mater. Interfaces, 2014, 6, 17059-17066.

41 A. Telecka, S. Murthy, L. Schneider, H. Pranov and R. Taboryski, ACS Macro Lett., 2016, 5, 1034-1038.

42 A. Steele, I. Bayer and E. Loth, Nano Lett., 2009, 9, 501-505. 43 T. S. Wong, S. H. Kang, S. K. Y. Tang, E. J. Smythe, B. D. Hatton, A. Grinthal and J. Aizenberg, Nature, 2011, 477, 443-447.

44 J. Drelich, E. Chibowski, D. D. Meng and K. Terpilowski, Soft Matter, 2011, 7, 9804-9828.

45 D. Quere, Annu. Rev. Mater. Res., 2008, 38, 71-99.

46 A. Tuteja, W. Choi, J. M. Mabry, G. H. McKinley and R. E. Cohen, Proc. Natl. Acad. Sci. U. S. A., 2008, 105, 18200-18205. 
47 A. Marmur, Langmuir, 2008, 24, 7573-7579.

48 M. Nosonovsky, Langmuir, 2007, 23, 3157-3161.

49 R. N. Wenzel, Ind. Eng. Chem., 1936, 28, 988-994.

50 A. Tuteja, W. Choi, M. L. Ma, J. M. Mabry, S. A. Mazzella, G. C. Rutledge, G. H. McKinley and R. E. Cohen, Science, 2007, 318, 1618-1622.

51 H. J. Butt, C. Semprebon, P. Papadopoulos, D. Vollmer, M. Brinkmann and M. Ciccotti, Soft Matter, 2013, 9, 418-428.

52 T. Jiang, Z. G. Guo and W. M. Liu, J. Mater. Chem. A, 2015, 3, 1811-1827.

53 H. Zhao and K. Y. Law, Langmuir, 2012, 28, 11821-11827.

54 P. Muthiah, B. Bhushan, K. Yun and H. Kondo, J. Colloid Interface Sci., 2013, 409, 227-236.

55 S. J. Pan, A. K. Kota, J. M. Mabry and A. Tuteja, J. Am. Chem. Soc., 2013, 135, 578-581.

56 H. J. Choi, S. Choo, J. H. Shin, K. I. Kim and H. Lee, J. Phys. Chem. C, 2013, 117, 24354-24359.

57 A. Susarrey-Arce, A. G. Marin, S. Schlautmann, L. Lefferts, J. G. E. Gardeniers and A. van Houselt, J. Micromech. Microeng., 2013, 23(2), 025004.

58 J. H. Kim, T. S. Shim and S. H. Kim, Adv. Mater., 2016, 28, 291-298.

59 M. Im, H. Im, J. H. Lee, J. B. Yoon and Y. K. Choi, Soft Matter, 2010, 6, 1401-1404.

60 S. Utech, K. Bley, J. Aizenberg and N. Vogel, J. Mater. Chem. A, 2016, 4, 6853-6859.

61 T. Darmanin, J. Tarrade, E. Celia and F. Guittard, J. Phys. Chem. C, 2014, 118, 2052-2057.

62 R. Campos, A. J. Guenthner, A. J. Meuler, A. Tuteja, R. E. Cohen, G. H. McKinley, T. S. Haddad and J. M. Mabry, Langmuir, 2012, 28, 9834-9841.

63 G. Hayase, K. Kanamori, G. Hasegawa, A. Maeno, H. Kaji and K. Nakanishi, Angew. Chem., Int. Ed., 2013, 52, 10788-10791. 64 Y. Li, X. T. Zhu, X. Y. Zhou, B. Ge, S. W. Chen and W. S. Wu, Appl. Phys. A: Mater. Sci. Process., 2014, 115, 765-770.

65 T. Li, K. Y. Wu, T. Rindzevicius, Z. L. Wang, L. Schulte, M. S. Schmidt, A. Boisen and S. Ndoni, ACS Appl. Mater. Interfaces, 2016, 8, 15668-15675.
66 T. Li, Z. L. Wang, L. Schulte, O. Hansen and S. Ndoni, RSC Adv., 2015, 5, 102619-102624.

67 J. B. Chang, H. K. Choi, A. F. Hannon, A. Alexander-Katz, C. A. Ross and K. K. Berggren, Nat. Commun., 2014, 5, 3305.

68 F. Ferrarese Lupi, T. J. Giammaria, F. G. Volpe, F. Lotto, G. Seguini, B. Pivac, M. Laus and M. Perego, ACS Appl. Mater. Interfaces, 2014, 6, 21389-21396.

69 A. Zeniou, K. Ellinas, A. Olziersky and E. Gogolides, Nanotechnology, 2014, 25(3), 035302.

70 A. Checco, A. Rahman and C. T. Black, Adv. Mater., 2014, 26, 886-891.

71 A. Checco, B. M. Ocko, A. Rahman, C. T. Black, M. Tasinkevych, A. Giacomello and S. Dietrich, Phys. Rev. Lett., 2014, 112(21), 216101.

72 S. Hoshian, V. Jokinen, V. Somerkivi, A. R. Lokanathan and S. Franssila, ACS Appl. Mater. Interfaces, 2015, 7, 941-949.

73 S. Lee, G. S. Jeong, J. Kim, J. Yoon, S. Han, J. Y. Kang, S. Chung and S. H. Lee, Microsyst. Technol., 2013, 19, 10251032.

74 L. C. Gao and T. J. McCarthy, Langmuir, 2007, 23, 3762-3765. 75 R. N. Wenzel, Ind. Eng. Chem., 1936, 28, 988-994.

76 K. Wu, T. Li, M. S. Schmidt, T. Rindzevicius, A. Boisen and S. Ndoni, Adv. Funct. Mater., 2018, 28, 1704818.

77 A. K. Kota, G. Kwon and A. Tuteja, NPG Asia Mater., 2014, 6, e109.

78 N. K. Mandsberg and T. Rafael, Surf. Topogr.: Metrol. Prop., 2017, 5, 024001.

79 K. Y. Yeh, K. H. Cho, Y. H. Yeh, A. Promraksa, C. H. Huang, C. C. Hsu and L. J. Chen, Nanotechnology, 2014, 25(34), 345303.

80 D. Ebert and B. Bhushan, J. Colloid Interface Sci., 2012, 384, 182-188.

81 B. Bhushan and M. Nosonovsky, Philos. Trans. R. Soc., A, 2010, 368, 4713-4728.

82 Y. H. Xiu, Y. Liu, D. W. Hess and C. P. Wong, Nanotechnology, 2010, 21(15), 155705.

83 S. Ndoni, P. Jannasch, N. B. Larsen and K. Almdal, Langmuir, 1999, 15, 3859-3865. 